\title{
Linear Algebra and Differential Calculus in Pseudo-Intervals Vector Space
}

\author{
A. KENOUFI \\ Received on October 13, 2014 / Accepted on August 30, 2016
}

\begin{abstract}
In this paper one proposes to use a new approach of interval arithmetic, the so-called pseudointervals $[1,5,13]$. It uses a construction which is more canonical and based on the semi-group completion into the group, and it allows to build a Banach vector space. This is achieved by embedding the vector space into free algebra of dimensions higher than 4. It permits to perform linear algebra and differential calculus with pseudo-intervals. Some numerical applications for interval matrix eigenmode calculation, inversion and function minimization are exhibited for simple examples.
\end{abstract}

Keywords: Pseudo-intervals arithmetic, free algebra, inclusion function, linear algebra, differential calculus, optimization.

\section{INTRODUCTION}

Intervals are mathematical objects which allow to describe quantities with uncertainity due to the lack of knowledge of the studied phenomenon or experimental measurement accuracy. This is common in natural sciences and engineering. Even if nowadays we consider R.E. Moore [20, $21,22,29,30]$ as the first mathematician who has proposed a rigorous framework for interval computations, the famous Archimedes from Syracuse (287-212 b.C) proposed 23 centuries before a two-sides bounding of $\pi: 3+\frac{10}{71}<\pi<3+\frac{1}{7}$.

Interval arithmetic, and interval analysis (IA) have been introduced as a computing framework which allows to perform analysis by computing mathematic bounds. Its extensions of the areas in applied and computational mathematics are important: non-linear problems, partial differential equations, inverse problems, global optimization and set inversion [1, 13, 14, 20, 21, 22, 30, 32]. It finds a large place of applications in controllability, automation, robotics, embedded systems, biomedical, haptic interfaces, form optimization, analysis of architecture plans, etc.

Several softwares and computational libraries to perform calculations with intervals such as INTLAB [7], INTOPT90, GLOBSOL [12], and Numerica [27] have been developed those last decades. But, due to the lack of distributivity of IA, their Achille's heel remains the construction of the inclusion function from the natural one. Some approaches using boolean inclusion 
tests, series or limited expansions of the natural function where the derivatives are computed at a certain point of the intervals, have been developed to circumvent this problem [1, 13, 14]. Nevertheless, those transfers from real functions to functions defined on intervals are not systematic and not given by a formal process. This yields to the fact that the inclusion function definition has to be adapted to each problem with the risk to miss the primitive scope. Moreover, linear algebra and differential calculus need to be performed in the framework of vector space theory and not within semi-group one.

This article reminds first in Section 2 the definition and characteristics of the intervals semigroup $\mathbb{I R}$ and the construction of its associated vector space $\overline{\mathbb{I R}}$, and how to get an associative and distributive arithmetic of intervals, called pseudo-intervals arithmetic, by embedding the vector space into a free algebra with dimension 4, 5, or 7 [1, 5, 13]. A systematic way to build inclusion functions, i.e. interval-valued functions defined on $\overline{\mathbb{R}}$, is presented in Section 2. In Section 3, one shows that $\overline{\mathbb{I}}$ can be endowed with a Banach space topology and allows differential calculus. Since a new algorithm for set inversion within pseudo-intervals framework has already been presented in previous papers [1, 13], only examples of interval matrix eigenmode calculation, inversion, and minimization of interval functions are exhibited in each section. We have choosen python programming langage [28] to implement pseudo-intervals arithmetic. The main reason is that it is a free object-oriented langage, with a huge number of numerical libraries. Another advantage of python is that first, it is possible to link the source code with others written in C/C++, FORTRAN, and second, it interacts easily with other calculations tools such as SAGE [31] and Maxima [19] in order to perform formal calculations. But here, one presents pure numerical applications in python.

\section{AN ALGEBRAIC APPROACH FOR INTERVALS}

An interval $X$ is defined as a non-empty, closed and connected set of real numbers. One writes real numbers as intervals with same bounds, $\forall a \in \mathbb{R}, a \equiv[a, a]$. We denote by $\mathbb{R}=\mathcal{P}_{1}$ the set of intervals of $\mathbb{R}$. The arithmetic operations on intervals, called Minkowski or classical operations, are defined such that the result of the corresponding operation on elements belonging to operand intervals belongs to the resulting interval. That is, if $\diamond \in\{+,-, *, /\}$ denotes one of the usual operations, one has, if $X$ and $Y$ are bounded intervals of $\mathbb{R}$,

$$
X \diamond Y=\{x \diamond y / x \in X, y \in Y\}
$$

Although, $\overline{\mathbb{I}}$ is provided with a pseudo-inverse operation, it does not satisfy $X-X=0$, and hence a substraction in the usual sense cannot be obtained. In many problems using interval arithmetic, that is the set $\mathbb{R}$ with the Minkowski operations, there exists an informal transfers principle which allows, to associate with a real function $f$ a function define on the set of intervals $\mathbb{I R}$ which coincides with $f$ on the interval reduced to a point. But this transferred function is not unique. For example, if we consider the real function $f(x)=x^{2}+x=x(x+1)$, we associate naturally the functions $\widetilde{f}_{1}, \widetilde{f}_{2}: \mathbb{R} \longrightarrow \mathbb{R} \mathbb{R}$ given by $\widetilde{f}_{1}(X)=X(X+1)$ and $\widetilde{f}_{2}(X)=X^{2}+X$. These two functions do not coincide. Usually this problem is removed considering the most 
interesting transfers. But the qualitative "interesting" depends of the studied model and it is not given by a formal process.

In this paper, we determine a natural extension $\overline{\mathbb{I R}}$ of $\mathbb{I R}$ provided with a vector space structure. The vectorial substraction $X \backslash Y$ does not correspond to the semantical difference of intervals and the interval $\backslash X$ has no real interpretation. But these anti-intervals have a computational role.

An algebraic extension of the classical interval arithmetic, called generalized interval arithmetic [21, 32] has been proposed first by M. Warmus [33, 34]. It has been followed in the seventies by H.J. Ortolf and E. Kaucher [8, 9, 10, 11, 24]. In this former interval arithmetic, the intervals form a group with respect to addition and a complete lattice with respect to inclusion. In order to adapt it to semantic problems, Gardenes et al. have developed an approach called modal interval arithmetic [2, 3, 4]. S. Markov and others investigate the relation between generalized intervals operations and Minkowski operations for classic intervals and propose the so-called directed interval arithmetic, in which Kaucher's generalized intervals can be viewed as classic intervals plus direction, hence the name directed interval arithmetic $[15,16]$. In this arithmetic framework, proper and improper intervals are considered as intervals with sign [17]. Interesting relations and developments for proper and improper intervals arithmetic and for applications can be found in literature $[23,26,25]$.

Our approach $[1,5,13]$, that we remind below in this article, is similar to the previous ones in the sense that intervals are extended to generalized intervals; intervals and anti-intervals correspond respectively to the proper and improper ones. However one uses a construction which is more canonical and based on the semi-group completion into a group, which allows then to build the associated real vector space, and to get an analogy with directed intervals.

\subsection{The real vector space $\overline{\mathbb{I}}$}

Let $\mathbb{I R}$ be the set of intervals. It is in one to one correspondence and can be represented as a point in the half-plane of $\mathbb{R}^{2}, \mathcal{P}_{1}=\left\{(a, b) \in \mathbb{R}^{2}, a \leq b\right\}$. The set $\mathcal{P}_{2}=\left\{(a, b) \in \mathbb{R}^{2}, a \geq b\right\}$ is the set of anti-intervals. $\mathbb{I R}$ is closed for the addition and endowed with a regular semi-group structure. The multiplication $*$ is not globally defined.

We recall briefly the construction proposed by Markov [18] to define a structure of abelian group. As $(\mathbb{R},+)$ is a commutative and regular semi-group, the quotient set, denoted by $\overline{\mathbb{I}}$, associated with the equivalence relations:

$$
(A, B) \sim(C, D) \Longleftrightarrow A+D=B+C,
$$

for all $A, B, C, D \in \mathbb{I}$, is provided with a structure of abelian group for the natural addition:

$$
\overline{(A, B)}+\overline{(C, D)}=\overline{(A+C, B+D)}
$$

where $\overline{(A, B)}$ is the equivalence class of $(A, B)$. We denote by $\backslash \overline{(A, B)}$ the inverse of $\overline{(A, B)}$ for the interval addition. 
We have $\backslash \overline{(A, B)}=\overline{(B, A)}$. If $X=[a, a], a \in \mathbb{R}$, then $\overline{(X, 0)}=\overline{(0,-X)}$ where $-X=$ $[-a,-a]$, and $\backslash \overline{(X, 0)}=\overline{(0, X)}$. In this case, we identify $X=[a, a]$ with $a$ and we denote always by $\mathbb{R}$ the subset of intervals of type $[a, a]$.

Naturally, the group $\overline{\mathbb{I R}}$ is isomorphic to the additive group $\mathbb{R}^{2}$ by the isomorphism $\overline{(([a, b],[c, d])} \rightarrow(a-c, b-d)$. We find the notion of generalized interval and this yields immediately to the following result:

Proposition 1. Let $\mathcal{X}=\overline{(X, Y)} \in \overline{\mathbb{I R}}$, and $l: \mathbb{R} \longmapsto \mathbb{R}$ which gives the interval length. Thus

1. If $l(Y)<l(X)$, there is an unique $A \in \mathbb{R} \backslash \mathbb{R}$ such that $\chi=\overline{(A, 0)}$,

2. If $l(Y)>l(X)$, there is an unique $A \in \mathbb{R} \backslash \mathbb{R}$ such that $\chi=\overline{(0, A)}=\backslash \overline{(A, 0)}$,

3. If $l(Y)=l(X)$, there is an unique $A=\alpha \in \mathbb{R}$ such that $\chi=\overline{(\alpha, 0)}=\overline{(0,-\alpha)}$.

Any element $\mathcal{X}=\overline{(A, 0)}$ with $A \in \mathbb{R}-\mathbb{R}$ is said positive and we write $\mathcal{X}>0$. Any element $\chi=\overline{(0, A)}$ with $A \in \mathbb{R}-\mathbb{R}$ is said negative and we write $\mathcal{X}<0$. We write $\chi \geq \mathcal{X}^{\prime}$ if

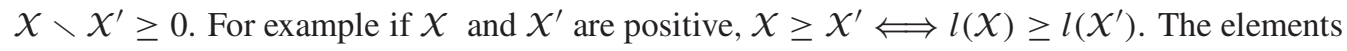
$\overline{(\alpha, 0)}$ with $\alpha \in \mathbb{R}^{*}$ are neither positive nor negative.

In [18], one defines on the abelian group $\overline{\mathbb{I R}}$, a structure of quasi linear space. Our approach is a little bit different. We propose to construct a real vector space structure. We consider the external multiplication:

$$
\cdot: \mathbb{R} \times \overline{\mathbb{I R}} \longrightarrow \overline{\mathbb{I R}}
$$

defined, for all $A \in \mathbb{R}$, by

$$
\left\{\begin{array}{l}
\alpha \cdot \overline{(A, 0)}=\overline{(\alpha A, 0)}, \\
\alpha \cdot \overline{(0, A)}=\overline{(0, \alpha A),}
\end{array}\right.
$$

for all $\alpha>0$. If $\alpha<0$ we put $\beta=-\alpha$. So we put:

$$
\left\{\begin{array}{l}
\alpha \cdot \overline{(A, 0)}=\overline{(0, \beta A)}, \\
\alpha \cdot \overline{(0, A)}=\overline{(\beta A, 0)} .
\end{array}\right.
$$

We denote $\alpha \mathcal{X}$ instead of $\alpha \cdot \mathcal{X}$. This operation satisfies

1. For any $\alpha \in \mathbb{R}$ and $\chi \in \overline{\mathbb{I R}}$ we have:

$$
\left\{\begin{array}{l}
\alpha(\backslash \mathcal{X})=\backslash(\alpha \mathcal{X}), \\
(-\alpha) \mathcal{X}=\backslash(\alpha \mathcal{X})
\end{array}\right.
$$

2. For all $\alpha, \beta \in \mathbb{R}$, and for all $\mathcal{X}, \mathcal{X}^{\prime} \in \overline{\mathbb{I}}$, we have

$$
\left\{\begin{array}{l}
(\alpha+\beta) \mathcal{X}=\alpha \mathcal{X}+\beta \mathcal{X} \\
\alpha\left(\mathcal{X}+\mathcal{X}^{\prime}\right)=\alpha \mathcal{X}+\alpha \mathcal{X}^{\prime} \\
(\alpha \beta) \mathcal{X}=\alpha(\beta \mathcal{X})
\end{array}\right.
$$


Theorem 1. The triplet $(\overline{\mathbb{R}},+, \cdot)$ is a real vector space and the vectors $\chi_{1}=\overline{([0,1], 0)}$ and $\chi_{2}=\overline{([1,1], 0)}$ of $\overline{\mathbb{I R}}$ determine a basis of $\overline{\mathbb{I}}$. So $\operatorname{dim}_{\mathbb{R}} \overline{\mathbb{I}}=2$.

Proof. We have the following decompositions:

$$
\left\{\begin{array}{l}
\overline{([a, b], 0)}=(b-a) \chi_{1}+a \chi_{2} \\
\overline{(0,[c, d])}=(c-d) \chi_{1}-c \mathcal{X}_{2}
\end{array}\right.
$$

The linear map

$$
\varphi: \overline{\mathbb{I R}} \longrightarrow \mathbb{R}^{2}
$$

defined by

$$
\left\{\begin{array}{l}
\varphi(\overline{([a, b], 0)})=(b-a, a), \\
\varphi(\overline{(0,[c, d])})=(c-d,-c)
\end{array}\right.
$$

is a linear isomorphism and $\overline{\mathbb{I}}$ is canonically isomorphic to $\mathbb{R}^{2}$.

Definition 1. $(\overline{\mathbb{I R}},+, \cdot)$ is called the vector space of pseudo-intervals.

\subsection{A 4-dimensional free algebra associated with $\overline{\mathbb{I R}}$}

Consider the following subset of $\mathcal{P}_{1}$ :

$$
\left\{\begin{array}{l}
\mathcal{P}_{1,1}=\left\{(a, b) \in \mathcal{P}_{1}, a \geq 0, b \geq 0\right\}, \\
\mathcal{P}_{1,2}=\left\{(a, b) \in \mathcal{P}_{1}, a \leq 0, b \geq 0\right\}, \\
\mathcal{P}_{1,3}=\left\{(a, b) \in \mathcal{P}_{1}, a \leq 0, b \leq 0\right\}
\end{array}\right.
$$

One observes that the semi-group $\mathbb{I R}$ can be identified to $\mathcal{P}_{1,1} \cup \mathcal{P}_{1,2} \cup \mathcal{P}_{1,3}$. Let us consider as well the following vectors of $\mathbb{R}^{2}$

$$
\left\{\begin{array}{l}
e_{1}=(1,1) \\
e_{2}=(0,1) \\
e_{3}=(-1,0) \\
e_{4}=(-1,-1)
\end{array}\right.
$$

They correspond to the intervals [1, 1], [0,1], [-1, 0], and [-1, -1]. Any point of $\mathcal{P}_{1,1} \cup \mathcal{P}_{1,2} \cup$ $\mathcal{P}_{1,3}$ admits the decomposition

$$
(a, b)=\alpha_{1} e_{1}+\alpha_{2} e_{2}+\alpha_{3} e_{3}+\alpha_{4} e_{4}
$$

with $\alpha_{i} \geq 0$. The dependance relations between the vectors $e_{i}$ are

$$
\left\{\begin{array}{l}
e_{2}=e_{3}+e_{1} \\
e_{4}=-e_{1}
\end{array}\right.
$$

Thus there exists a unique decomposition of $(a, b)$ in a chosen basis such that the coefficients are non negative. These basis are $\left\{e_{1}, e_{2}\right\}$ for $\mathcal{P}_{1,1},\left\{e_{2}, e_{3}\right\}$ for $\mathcal{P}_{1,2},\left\{e_{3}, e_{4}\right\}$ for $\mathcal{P}_{1,3}$. Let us consider 
the free algebra of basis $\left\{e_{1}, e_{2}, e_{3}, e_{4}\right\}$ whose products correspond to the Minkowski products. The multiplication table is

\begin{tabular}{|l|l|l|l|l|}
\hline & $e_{1}$ & $e_{2}$ & $e_{3}$ & $e_{4}$ \\
\hline$e_{1}$ & $e_{1}$ & $e_{2}$ & $e_{3}$ & $e_{4}$ \\
\hline$e_{2}$ & $e_{2}$ & $e_{2}$ & $e_{3}$ & $e_{3}$ \\
\hline$e_{3}$ & $e_{3}$ & $e_{3}$ & $e_{2}$ & $e_{2}$ \\
\hline$e_{4}$ & $e_{4}$ & $e_{3}$ & $e_{2}$ & $e_{1}$ \\
\hline
\end{tabular}

Definition 2. This algebra is associative and its elements are called pseudo-intervals.

\subsection{Pseudo-intervals product}

Let $\varphi: \overline{\mathbb{R}} \longrightarrow \mathcal{A}_{4}$ the natural injective embedding, $F \subset \mathcal{A}_{4}$ the linear subspace generated by $\left\{e_{1}-e_{2}+e_{3}, e_{1}+e_{4}\right\}, \psi$ the canonical embedding from $\mathcal{A}_{4}$ to $\mathcal{A}_{4} / F$ and $\varphi^{\prime}=\psi \circ \varphi[1,5,13]$. We identify an interval with its image in $\mathcal{A}_{4}$. The application $\varphi$ is not bijective. Its image on the elements $\mathcal{X}=\overline{(X, 0)}=\overline{([a, b], 0)}$ is

$$
\left\{\begin{array}{l}
X=[a, b] \in \mathcal{P}_{1,1}, \varphi(\mathcal{X})=a e_{1}+(b-a) e_{2} \quad(a \geq 0, b-a \geq 0) \\
X=[a, b] \in \mathcal{P}_{1,2}, \varphi(X)=-a e_{3}+b e_{2} \quad(-a \geq 0, b \geq 0) \\
X=[a, b] \in \mathcal{P}_{1,3}, \varphi(X)=-b e_{4}+(b-a) e_{3} \quad(-b \geq 0, b-a \geq 0)
\end{array}\right.
$$

Theorem 2. The multiplication

$$
\chi \bullet \mathcal{Y}=\bar{\varphi}^{-1}\left(\varphi^{\prime}(\chi) \cdot \varphi^{\prime}(\mathcal{Y})\right)
$$

is distributive with respect the addition.

Proof. Practically the multiplication of two intervals will so be made: let $X, Y \in \mathbb{R}$. Thus $X=\sum \alpha_{i} e_{i}, Y=\sum \beta_{i} e_{i}$ with $\alpha_{i}, \beta_{j} \geq 0$ and we have the product

$$
X \bullet Y=\bar{\varphi}^{-1}\left(\varphi^{\prime}(X) \cdot \varphi^{\prime}(Y)\right)
$$

this product is well defined because $\overline{\varphi^{\prime}(X) \cdot \varphi^{\prime}(Y)} \in \operatorname{Im} \bar{\varphi}$. This product is distributive because

$$
\begin{aligned}
X \bullet(Y+Z) & =\bar{\varphi}^{-1}\left(\varphi^{\prime}(X) \cdot \varphi^{\prime}(Y+Z)\right) \\
& =\bar{\varphi}^{-1}\left(\varphi^{\prime}(X) \cdot\left(\varphi^{\prime}(Y)+\varphi^{\prime}(Z)\right)\right. \\
& =\bar{\varphi}^{-1}\left(\varphi^{\prime}(X) \cdot \varphi^{\prime}(Y)+\varphi^{\prime}(X) \cdot \varphi^{\prime}(Z)\right) \\
& =X \bullet Y+X \bullet Z
\end{aligned}
$$

Remark. We have

$$
\left.\bar{\varphi}^{-1}\left(\varphi^{\prime}(X) \cdot \varphi^{\prime}(Y+Z)\right) \neq \bar{\varphi}^{-1}\left(\varphi^{\prime}(X)\right) \cdot \bar{\varphi}^{-1}\left(\varphi^{\prime}(Y+Z)\right)\right) .
$$


We shall be careful not to return in $\mathbb{I R}$ during the calculations as long as the result is not found. Otherwise we find the semantic problems of the distributivity.

We extend naturally the map $\varphi: \mathbb{I} \mathbb{R} \longrightarrow \mathcal{A}_{4}$ to $\overline{\mathbb{I}}$ by

$$
\left\{\begin{array}{l}
\varphi \overline{(A, 0)}=\varphi(A) \\
\varphi \overline{(0, A)}=-\varphi(A)
\end{array}\right.
$$

for every $A \in \mathbb{I}$.

\subsection{Pseudo-intervals division}

Division between intervals can also be defined with solving $X \cdot Y=(1,0,0,0)$ in $\mathcal{A}_{4}$ or in a isomorphic algebra $\mathcal{A}_{4}^{\prime}$. In $\mathcal{A}_{4}$ we consider the change of basis

$$
\left\{\begin{array}{l}
e_{1}^{\prime}=e_{1}-e_{2} \\
e_{i}^{\prime}=e_{i}, i=2,3 \\
e_{4}^{\prime}=e_{4}-e_{3} .
\end{array}\right.
$$

This change of basis shows that $\mathcal{A}_{4}$ is isomorphic to $\mathcal{A}_{4}^{\prime}$

\begin{tabular}{|l|l|l|l|l|}
\hline & $e_{1}$ & $e_{2}$ & $e_{3}$ & $e_{4}$ \\
\hline$e_{1}$ & $e_{1}$ & 0 & 0 & $e_{4}$ \\
\hline$e_{2}$ & 0 & $e_{2}$ & $e_{3}$ & 0 \\
\hline$e_{3}$ & 0 & $e_{3}$ & $e_{2}$ & 0 \\
\hline$e_{4}$ & $e_{4}$ & 0 & 0 & $e_{1}$ \\
\hline
\end{tabular}

The unit of $\mathcal{A}_{4}^{\prime}$ is the vector $e_{1}+e_{2}$. This algebra is a direct sum of two ideals: $\mathcal{A}_{4}^{\prime}=I_{1}+I_{2}$ where $I_{1}$ is generated by $e_{1}$ and $e_{4}$ and $I_{2}$ is generated by $e_{2}$ and $e_{3}$. It is not an integral domain, that is, we have divisors of 0 . For example $e_{1} \cdot e_{2}=0$.

Proposition 2. The multiplicative group $\mathcal{A}_{4}^{*}$ of invertible elements of $\mathcal{A}_{4}$ is the set of elements $x=\left(x_{1}, x_{2}, x_{3}, x_{4}\right)$ such that

$$
\left\{\begin{array}{l}
x_{4} \neq \pm x_{1} \\
x_{3} \neq \pm x_{2}
\end{array}\right.
$$

This means that the invertible intervals do not contain 0 . If $x \in \mathcal{A}_{4}^{*}$ we have:

$$
x^{-1}=\left(\frac{x_{1}}{x_{1}^{2}-x_{4}^{2}}, \frac{x_{2}}{x_{2}^{2}-x_{3}^{2}}, \frac{x_{3}}{x_{2}^{2}-x_{3}^{2}}, \frac{x_{4}}{x_{1}^{2}-x_{4}^{2}}\right) \text {. }
$$

\subsection{Pseudo-intervals monotonicity property}

Let us compute the product of pseudo-intervals using the product in $\mathcal{A}_{4}$ and compare it with the Minkowski product. Let $X=[a, b]$ and $Y=[c, d]$ two intervals.

Lemma 1. If $X$ and $Y$ are not in the same piece $\mathcal{P}_{1, i}$, then $X \bullet Y$ corresponds to the Minkowski product. 
Proof. i) If $X \in \mathcal{P}_{1,1}$ and $Y \in \mathcal{P}_{1,2}$ then $\varphi(X)=(a, b-a, 0,0)$ and $\varphi(Y)=(0, d,-c, 0)$. Thus

$$
\begin{aligned}
\varphi(X) \varphi(Y) & =\left(a e_{1}+(b-a) e_{2}\right)\left(d e_{2}-c e_{3}\right) \\
& =b d e_{2}-c b e_{3} \\
& =(0, b d,-c b, 0) \\
& =\varphi([c b, b d]) .
\end{aligned}
$$

ii) If $X \in \mathcal{P}_{1,1}$ and $Y \in \mathcal{P}_{1,3}$ then $\varphi(X)=(a, b-a, 0,0)$ and $\varphi(Y)=(0,0, d-c,-d)$. Thus

$$
\begin{aligned}
\varphi(X) \varphi(Y) & =\left(a e_{1}+(b-a) e_{2}\right)\left((d-c) e_{3}-d e_{4}\right) \\
& =(a d-b c) e_{3}-a d e_{4} \\
& =(0,0, a d-c b,-a d) \\
& =\varphi([b c, a d])
\end{aligned}
$$

iii) If $X \in \mathcal{P}_{1,2}$ and $Y \in \mathcal{P}_{1,3}$ then $\varphi(X)=(0, b,-a, 0)$ and $\varphi(Y)=(0,0, d-c,-d)$. Thus

$$
\begin{aligned}
\varphi(X) \varphi(Y) & =\left(b e_{2}-a e_{3}\right)\left((d-c) e_{3}-d e_{4}\right) \\
& =a c e_{2}-b c e_{3} \\
& =(0, a c,-c b, 0) \\
& =\varphi([b c, a d]) .
\end{aligned}
$$

Lemma 2. If $X$ an $Y$ are both in the same piece $\mathcal{P}_{1,1}$ or $\mathcal{P}_{1,3}$, then the product $X \bullet Y$ corresponds to the Minkowski product.

The proof is analogous to the previous.

Let us assume that $X=[a, b]$ and $Y=[c, d]$ belong to $\mathcal{P}_{1,2}$. Thus $\varphi(X)=(0, b,-a, 0)$ and $\varphi(Y)=(0, d,-c, 0)$. We obtain

$$
X Y=\left(b e_{2}-a e_{3}\right)\left(d e_{2}-c e_{3}\right)=(b d+a c) e_{2}+(-b c-a d) e_{3} .
$$

Thus

$$
[a, b][c, d]=[b c+a d, b d+a c] .
$$

This result is greater that all the possible results associated with the Minkowski product. However, we have the following property:

Proposition 3. Monotony property: Let $\chi_{1}, \chi_{2} \in \overline{\mathbb{R}}$. Then

$$
\left\{\begin{array}{l}
\chi_{1} \subset \chi_{2} \Longrightarrow \chi_{1} \bullet Z \subset \chi_{2} \bullet Z \text { for all } Z \in \overline{\mathbb{R}} \\
\bar{\varphi}\left(\chi_{1}\right) \leq \bar{\varphi}\left(\chi_{2}\right) \Longrightarrow \bar{\varphi}\left(\chi_{1} \bullet Z\right) \leq \bar{\varphi}\left(\chi_{2} \bullet Z\right)
\end{array}\right.
$$

The order relation on $\mathcal{A}_{4}$ that ones uses here is

$$
\left\{\begin{array}{l}
\left(x_{1}, x_{2}, 0,0\right) \leq\left(y_{1}, y_{2}, 0,0\right) \Longleftrightarrow y_{1} \leq x_{1} \text { and } x_{2} \leq y_{2}, \\
\left(x_{1}, x_{2}, 0,0\right) \leq\left(0, y_{2}, y_{3}, 0\right) \Longleftrightarrow x_{2} \leq y_{2}, \\
\left(0, x_{2}, x_{3}, 0\right) \leq\left(0, y_{2}, y_{3}, 0\right) \Longleftrightarrow x_{3} \leq y_{3} \text { and } x_{2} \leq y_{2}, \\
\left(0,0, x_{3}, x_{4}\right) \leq\left(0, y_{2}, y_{3}, 0\right) \Longleftrightarrow x_{3} \leq y_{3}, \\
\left(0,0, x_{3}, x_{4}\right) \leq\left(0,0, y_{3}, y_{4}\right) \Longleftrightarrow x_{3} \leq y_{3} \text { and } y_{4} \leq x_{4}
\end{array}\right.
$$


Proof. Let us note that the second property is equivalent to the first. It is its translation in $\overline{\mathcal{A}_{4}}$. We can suppose that $\mathcal{X}_{1}$ and $\mathcal{X}_{2}$ are intervals belonging moreover to $\mathcal{P}_{1,2}: \varphi\left(\mathcal{X}_{1}\right)=$ $(0, b,-a, 0), \varphi\left(\mathcal{X}_{2}\right)=(0, d,-c, 0)$. If $\varphi(\mathcal{Z})=\left(z_{1}, z_{2}, z_{3}, z_{4}\right)$, then

$$
\left\{\begin{array}{l}
\bar{\varphi}\left(\mathcal{X}_{1} \bullet Z\right)=\left(0, b z_{1}+b z_{2}-a z_{3}-a z_{4},-a z_{1}+b z_{3}-a z_{2}+b z_{4}, 0\right) \\
\bar{\varphi}\left(\mathcal{X}_{2} \bullet Z\right)=\left(0, d z_{1}+d z_{2}-c z_{3}-c z_{4},-c z_{1}+d z_{3}-c z_{2}+d z_{4}, 0\right)
\end{array}\right.
$$

Thus

$$
\bar{\varphi}\left(\mathcal{X}_{1} \bullet \mathcal{Z}\right) \leq \bar{\varphi}\left(\mathcal{X}_{2} \bullet Z\right) \Longleftrightarrow\left\{\begin{array}{l}
(b-d)\left(z_{1}+z_{2}\right)-(a-c)\left(z_{3}-z_{4}\right) \leq 0 \\
-(a-c)\left(z_{1}+z_{2}\right)+(b-d)\left(z_{3}=z_{4}\right) \leq 0
\end{array}\right.
$$

But $(b-d),-(a-c) \leq 0$ and $z_{2}, z_{3} \geq 0$. This implies $\bar{\varphi}\left(\mathcal{X}_{1} \bullet \mathcal{Z}\right) \leq \bar{\varphi}\left(\mathcal{X}_{2} \bullet \mathcal{Z}\right)$.

\subsection{The algebras $\mathcal{A}_{n}$ and a better result of the product}

We can refine the product to become closer to Minkowski's one. Consider the one dimensional extension $\mathcal{A}_{4} \oplus \mathbb{R} e_{5}=\mathcal{A}_{5}$, where $e_{5}$ is a vector corresponding to the interval $[-1,1]$ of $\mathcal{P}_{1,2}$. The multiplication table of $\mathcal{A}_{5}$ is

\begin{tabular}{|l|l|l|l|l|l|}
\hline & $e_{1}$ & $e_{2}$ & $e_{3}$ & $e_{4}$ & $e_{5}$ \\
\hline$e_{1}$ & $e_{1}$ & $e_{2}$ & $e_{3}$ & $e_{4}$ & $e_{5}$ \\
\hline$e_{2}$ & $e_{2}$ & $e_{2}$ & $e_{3}$ & $e_{3}$ & $e_{5}$ \\
\hline$e_{3}$ & $e_{3}$ & $e_{3}$ & $e_{2}$ & $e_{2}$ & $e_{5}$ \\
\hline$e_{4}$ & $e_{4}$ & $e_{3}$ & $e_{2}$ & $e_{1}$ & $e_{5}$ \\
\hline$e_{5}$ & $e_{5}$ & $e_{5}$ & $e_{5}$ & $e_{5}$ & $e_{5}$ \\
\hline
\end{tabular}

The piece $\mathcal{P}_{1,2}$ is written $\mathcal{P}_{1,2}=\mathcal{P}_{1,2,1} \cup \mathcal{P}_{1,2,1}$ where $\mathcal{P}_{1,2,1}=\{[a, b],-a \leq b\}$ and $\mathcal{P}_{1,2,2}=$ $\{[a, b],-a \geq b\}$. If $X=[a, b] \in \mathcal{P}_{1,2,1}$ and $Y=[c, d] \in \mathcal{P}_{1,2,2}$, thus

$\varphi(X) \cdot \varphi(Y)=(0, b+a, 0,0,-a) \cdot(0,0,-c-d, 0, d)=(0,-(a+b)(c+d), 0,0, a(c+d)+b d)$.

Thus we have

$$
X \bullet Y=[-b d-a c-a d,-b c]
$$

Example. Let $X=[-2,3]$ and $Y=[-4,2]$. We have $X \in \mathcal{P}_{1,2,1}$ and $Y \in \mathcal{P}_{1,2,2}$. The product in $\mathcal{A}_{4}$ gives

$$
X \bullet Y=[-16,14] .
$$

The product in $\mathcal{A}_{5}$ gives

$$
X \bullet Y=[-12,10] .
$$

The Minkowski product is

$$
[-2,3] \cdot[-4,2]=[-12,8]
$$


Thus the product in $\mathcal{A}_{5}$ is better than the product in $\mathcal{A}_{4}$ with respect to the partial order. Let's go further. Considering a partition of $\mathcal{P}_{1,2}$, we can define an extension of $\mathcal{A}_{4}$ of dimension $n$, the choice of $n$ depends on the approach wanted of the Minkowski product. For example, let us consider the vector $e_{6}$ corresponding to the interval $\left[-1, \frac{1}{2}\right]$. Thus the Minkowsky product gives $e_{6} \cdot e_{6}=e_{7}$ where $e_{7}$ corresponds to $\left[-\frac{1}{2}, 1\right]$. This yields to the fact that $\mathcal{A}_{6}$ is not an associative algebra but it is the case for $\mathcal{A}_{7}$ whose table of multiplication is

\begin{tabular}{|l|l|l|l|l|l|l|l|}
\hline & $e_{1}$ & $e_{2}$ & $e_{3}$ & $e_{4}$ & $e_{5}$ & $e_{6}$ & $e_{7}$ \\
\hline$e_{1}$ & $e_{1}$ & $e_{2}$ & $e_{3}$ & $e_{4}$ & $e_{5}$ & $e_{6}$ & $e_{7}$ \\
\hline$e_{2}$ & $e_{2}$ & $e_{2}$ & $e_{3}$ & $e_{3}$ & $e_{5}$ & $e_{6}$ & $e_{7}$ \\
\hline$e_{3}$ & $e_{3}$ & $e_{3}$ & $e_{2}$ & $e_{2}$ & $e_{5}$ & $e_{7}$ & $e_{6}$ \\
\hline$e_{4}$ & $e_{4}$ & $e_{3}$ & $e_{2}$ & $e_{1}$ & $e_{5}$ & $e_{7}$ & $e_{6}$ \\
\hline$e_{5}$ & $e_{5}$ & $e_{5}$ & $e_{5}$ & $e_{5}$ & $e_{5}$ & $e_{5}$ & $e_{5}$ \\
\hline$e_{6}$ & $e_{6}$ & $e_{6}$ & $e_{7}$ & $e_{7}$ & $e_{5}$ & $e_{7}$ & $e_{6}$ \\
\hline$e_{7}$ & $e_{7}$ & $e_{7}$ & $e_{6}$ & $e_{6}$ & $e_{5}$ & $e_{6}$ & $e_{7}$ \\
\hline
\end{tabular}

Example. Let $X=[-2,3]$ and $Y=[-4,2]$. The decomposition on the basis $\left\{e_{1}, \ldots, e_{7}\right\}$ with positive coefficients writes

$$
\begin{gathered}
X=e_{5}+2 e_{7}, \quad Y=2 e_{6} . \\
X \bullet Y=\left(e_{5}+2 e_{7}\right)\left(4 e_{6}\right)=4 e_{5}+8 e_{6}=[-12,8] .
\end{gathered}
$$

We obtain now the Minkowski product.

Remark 1. Pseudo-intervals division can be defined as well in $\mathcal{A}_{5}$ and $\mathcal{A}_{7}$.

\subsection{Inclusion functions}

It is necessary for some problems to extend the definition of a function defined for real numbers $f: \mathbb{R} \rightarrow \mathbb{R}$ to function defined for intervals $[f]: \mathbb{R} \rightarrow \mathbb{R}$ such as $[f]([a, a])=f(a)$ for any $a \in \mathbb{R}$. It will be convenient to have the same formal expression for $f$ and $[f]$. Usually the lack of distributivity in Minkowski arithmetic does not give the possibility to get the same formal expressions. But with the pseudo-intervals arithmetic we have presented, there is no data dependency anymore and one can define easily inclusion functions from the natural one. For example, let's extend to intervals the real functions $f_{0}(x)=x^{2}-2 x+1, f_{1}(x)=(x-1)^{2}, f_{2}(x)=x(x-2)+1$. Usually, with the Minkwoski operations, the three expressions of this same function for the interval $X=[3,4]$ are $[f]_{0}(X)=[2,11],[f]_{1}(X)=[4,9]$ and $[f]_{2}(X)=[6,12]$. Data dependancy occurs when the variable appears more than once in the function expression. The deep reason of that is the lack of distributivity in Minkowski arithmetic. But within the arithmetic developed in $\mathcal{A}_{4}$ or higher dimension free algebras [5], this problem vanishes. For example: with $X=[3,4]$ and since $X \in \mathcal{P}_{11}$,

$$
\varphi(X)=(3,4-3,0,0)=(3,1,0,0)=3 e_{1}+e_{2} .
$$


Since $e_{1}=(1,0,0,0), e_{2}=(0,1,0,0)$ and with means of product table, one has

$$
\begin{aligned}
\varphi\left([f]_{0}(X)\right)=\left(3 e_{1}+e_{2}\right)^{2}-2\left(3 e_{1}+e_{2}\right)+1 \\
=9 e_{1}^{2}+2 \cdot 3 e_{1} e_{2}+e_{2}^{2}-2 \cdot 3 e_{1}-2 e_{2}+1 \\
=9 e_{1}+6 e_{2}+e_{2}-6 e_{1}-2 e_{2}+e_{1}=4 e_{1}+5 e_{2} \\
=\varphi([4,9]), \\
\qquad\left([f]_{1}(X)\right)=\left(3 e_{1}+e_{2}-1\right)^{2} \\
=\left(2 e_{1}+e_{2}\right)^{2} \\
=4 e_{1}^{2}+4 e_{1} e_{2}+e_{2}^{2} \\
=4 e_{1}+4 e_{2}+e_{2} \\
=4 e_{1}+5 e_{2} \\
=\varphi([4,9]),
\end{aligned}
$$

and

$$
\begin{aligned}
\varphi\left([f]_{2}(X)\right) & =\left(3 e_{1}+e_{2}\right) \cdot\left(3 e_{1}+e_{2}-2\right)+1 \\
& =9 e_{1}+3 e_{1} e_{2}-6 e_{1}+3 e 1 e_{2}+e_{2}^{2}-2 e_{2}+e_{1} \\
& =4 e_{1}+3 e_{2}+3 e_{2}+e_{2}-2 e_{2} \\
& =4 e_{1}+5 e_{2} \\
& =\varphi([4,9]) .
\end{aligned}
$$

Thus, $[f]_{0}(X)=[f]_{1}(X)=[f]_{2}(X)=[4,9]$ and the inclusion function is defined univoquely regardless the way to write the original one.

On the other hand, the construction of the inclusion function depends on the type of problem one deals with. If one aims to perform set inversion for example, it has to be done in the semi-group $\mathbb{I} \mathbb{R}$. But, the substraction is not defined in $\mathbb{I R}$. This problem can be circumvented by replacing it with an addition and a multiplication with the interval $e_{4}=[-1,-1]$. This maintains the associativity and distributivity of arithmetic and permits to introduce a pseudo-substraction. For example: if $f(x)=x^{2}-x=x(x-1)$ for real numbers, one defines $[f](X)=X^{2}+e_{4} \cdot X$. One reminds the product $[-1,-1] \cdot[a, b]$ is equal to $[-b,-a]$. Due to the fact that the arithmetic is now associative and distributive, one does not have data dependency anymore and $[f](X)=$ $X^{2}+e_{4} \cdot X=X \cdot\left(X+e_{4}\right)$. The last term corresponds to the transfer of $x(x-1)$. Division can be transferred to the semi-group in the same way by replacing $\frac{1}{x}=x^{-1}$ with $X^{e_{4}}$.

Taylor polynomial expansions, differential calculus and linear algebra operations are defined only in a vector space. Therefore the transfer for the vector space is done directly. This permits to get infinitesimal intervals with the substraction and to compute derivatives. This is of course not allowed and not possible into the semi-group. From $\mathbb{I R}$ to the vector space $\overline{\mathbb{I}}, f: x \longmapsto-x$ is 
transferred to $[f]: X \equiv \overline{(X, 0)} \mapsto \backslash \overline{(X, 0)} \equiv \backslash X$. This means that $[a, b]$ substraction is the antiinterval $[-a,-b]$ addition. One of the most important consequence is that it is possible to transfer some functions directly to the pseudo-intervals. For example, it is easy to prove analytically in $\overline{\mathbb{I R}}$ that $[\exp ](\overline{([a, b], 0)})=\overline{([\exp (a), \exp (b)], 0)}$ with means of Taylor expansion.

\subsection{Numerical linear algebra examples}

One uses the $\mathcal{A}_{7}$ pseudo-intervals arithmetic for simple examples of linear algebra computations where matrix elements are intervals.

\subsubsection{Interval matrix diagonalization}

As an example, we would like to compute the largest eigenmode of the matrix $M$ whose elements are intervals centered around a certain real number with a radius $\epsilon$ :

$$
M=\left(\begin{array}{ccc}
{[2-\epsilon, 2+\epsilon]} & {[6-\epsilon, 6+\epsilon]} & {[5-\epsilon, 5+\epsilon]} \\
{[6-\epsilon, 6+\epsilon]} & {[2-\epsilon, 2+\epsilon]} & {[8-\epsilon, 8+\epsilon]} \\
{[5-\epsilon, 5+\epsilon]} & {[8-\epsilon, 8+\epsilon]} & {[6-\epsilon, 6+\epsilon]}
\end{array}\right) .
$$

If one uses scilab to compute the spectrum of the previous matrix without radius $(\epsilon=0)$, the highest eigenvalue is approximatively 16.345903 and the corresponding eigenvector is $(0.4728057,0.5716783,0.6705510)$. In order to show that arithmetics and interval algebra developed above are robust and stable, let's try to compute the highest eigenvalue of an interval matrix. One uses here the iterate power method, which is very simple and constitute the basis of several powerful methods such as deflation and others.

The Figures 1 and 2 show clearly for different value of $\epsilon$ the stability of the multiplication, and the largest eigenmode is recovered when $\epsilon=0$. The other eigen modes can be computed with the deflation methods for example which consists to withdraw the direction spanned by the eigenvector associated to the highest eigenvalue to the matrix by constructing its projector and to do the same. Several methods are available and efficient to achieve that $[6,35]$. We have choosen to compute only the highest eigenvalue and its corresponding eigenvector in order to show simply the efficiency of our new arithmetic.

\subsubsection{Interval matrix inversion}

Let's define the matrix $X$ whose elements are intervals centered around a certain real number with a radius $\epsilon$ :

$$
X=\left(\begin{array}{ccc}
{[-2-\epsilon,-2+\epsilon]} & {[-7-\epsilon, 7+\epsilon]} & {[4-\epsilon, 4+\epsilon]} \\
{[5-\epsilon, 5+\epsilon]} & {[-1-\epsilon,-1+\epsilon]} & {[6-\epsilon, 6+\epsilon]} \\
{[9-\epsilon, 9+\epsilon]} & {[-8-\epsilon,-8+\epsilon]} & {[3-\epsilon, 3+\epsilon]}
\end{array}\right)
$$




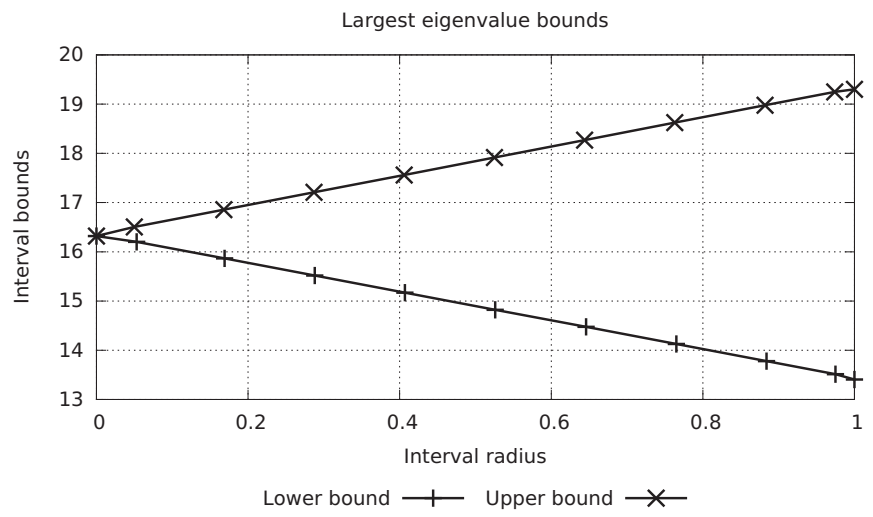

Figure 1: Largest eigenvalue convergence computed with iterate power method to the value computed with scilab 16.345903 .

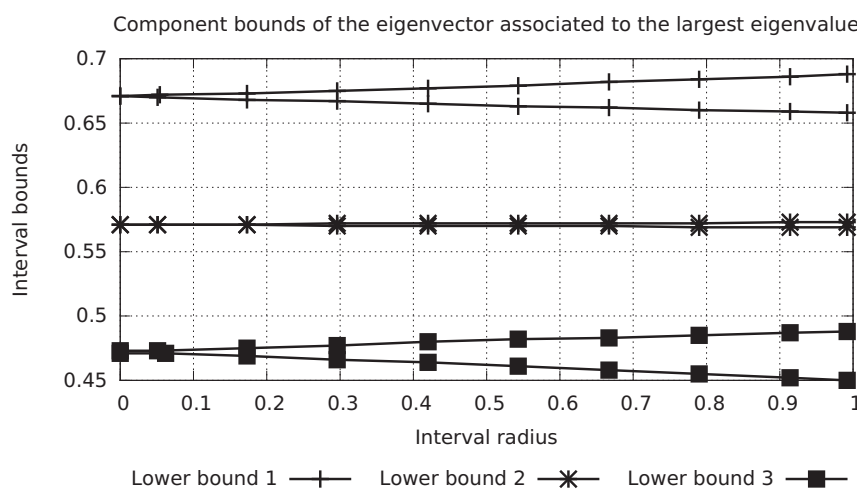

Figure 2: Eigenvector components associated to the largest eigenvalue convergence computed with iterate power method to the eigenvector computed with scilab $(0.4728057,0.5716783$, $0.6705510)$.

Scilab inversion function gives numerically for $\epsilon=0$

$$
X^{-1}=\left(\begin{array}{rrr}
-0.0924025 & 0.0225873 & 0.0780287 \\
-0.0800821 & 0.0862423 & -0.0657084 \\
0.0636550 & 0.1622177 & -0.0759754
\end{array}\right) .
$$

We would like to use the well-known Schutz-Hotelling algorithm $[6,35]$ to inverse the matrix $X$ since it uses simple arithmetic operations:

$$
X_{0}=\frac{X^{t}}{\sum_{i, j} A_{i j}^{2}}, X_{j}=X_{j-1}\left(2-A \cdot X_{j-1}\right), \forall n \geq 1
$$

The elements of inverse matrix columns are exhibited on Figures 3, 4, 5 and are converging to the right values when $\epsilon=0$. 


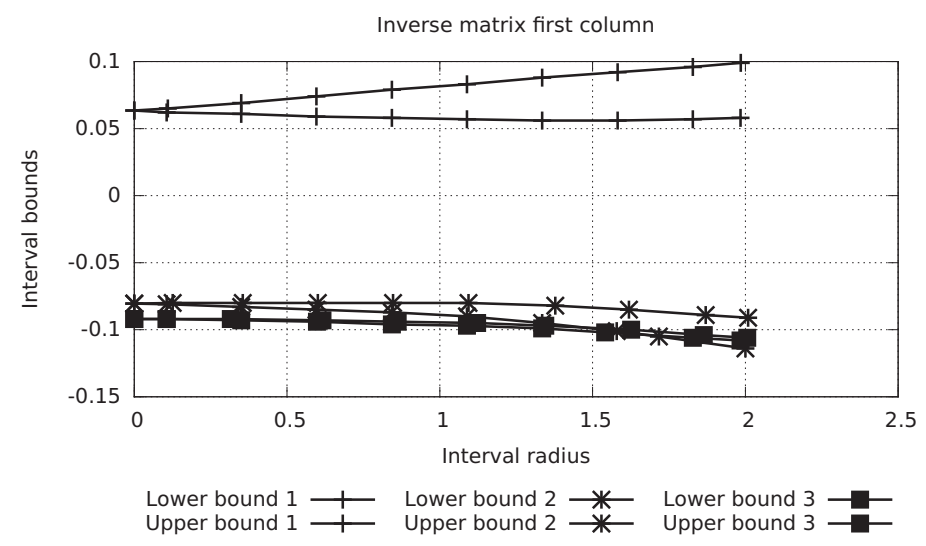

Figure 3: First column elements bounds convergence according interval radius $\epsilon$.

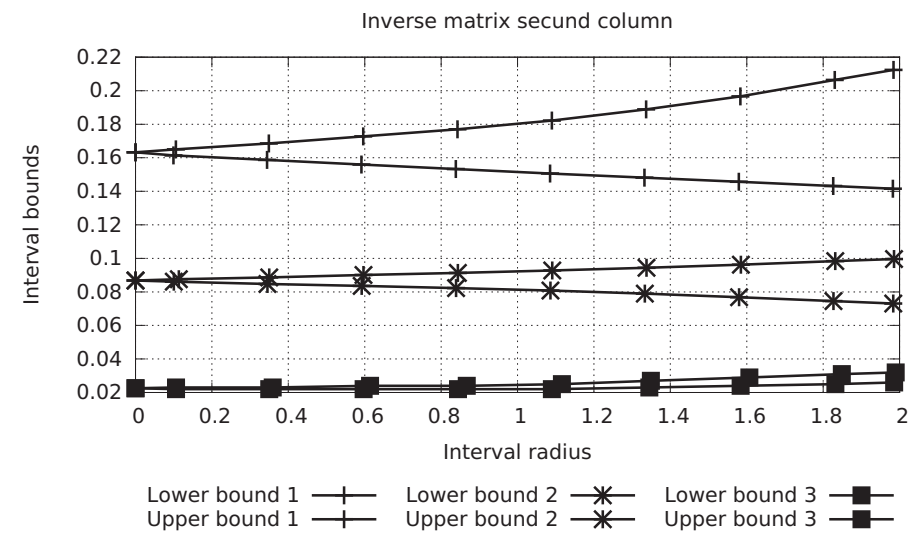

Figure 4: Second column elements bounds convergence according interval radius $\epsilon$.



Figure 5: Third column elements bounds convergence according interval radius $\epsilon$. 


\section{TOPOLOGY OF $\overline{\mathbb{I R}}$}

One shows in this section that $\overline{\mathbb{I}}$ can be endowed with the metric topology of a Banach space. This permits to define correctly continuity and differentiability of functions.

\subsection{Banach space structure of $\overline{\mathbb{I R}}$}

Any element $\mathcal{X} \in \overline{\mathbb{I R}}$ is written $\overline{(A, 0)}$ or $\overline{(0, A)}$. We define its length $l(\mathcal{X})$ as the length of $A$ and its center as $c(A)$ or $-c(A)$ in the second case.

Theorem 3. The map \|\|$: \overline{\mathbb{I R}} \longrightarrow \mathbb{R}^{+}$given by

$$
\|\chi\|=l(\mathcal{X})+|c(\mathcal{X})|
$$

for any $\chi \in \overline{\mathbb{I}}$ is a norm.

Proof. We have to verify the following axioms:

$$
\left\{\begin{array}{l}
\text { 1) }\|x\|=0 \Longleftrightarrow \chi=0, \\
\text { 2) } \forall \lambda \in \mathbb{R}\|\lambda \chi\|=|\lambda|\|\chi\|, \\
\text { 3) }\left\|x+\chi^{\prime}\right\| \leq\|\chi\|+\left\|\chi^{\prime}\right\| .
\end{array}\right.
$$

1) If $\|\mathcal{X}\|=0$, then $l(\mathcal{X})=|c(\mathcal{X})|=0$ and $\mathcal{X}=0$.

2) Let $\lambda \in \mathbb{R}$. We have

$$
\|\lambda \chi\|=l(\lambda \chi)+|c(\lambda \chi)|=|\lambda| \cdot l(X)+|\lambda| \cdot|c(\chi)|=|\lambda| \cdot|| \chi \| .
$$

3) One considers that $I$ refers to $X$ and $J$ refers to $\chi^{\prime}$ thus $\chi=\overline{(I, 0)}$ or $=\overline{(0, I)}$. We have to study the two different cases:

i) If $\mathcal{X}+\chi^{\prime}=\overline{(I+J, 0)}$ or $\overline{(0, I+J)}$, then

$$
\begin{aligned}
\left\|\chi+\mathcal{X}^{\prime}\right\| & =l(I+J)+|c(I+J)| \\
& =l(I)+l(J)+|c(I)+c(J)| \\
& \leq l(I)+|c(I)|+l(J)+|c(J)| \\
& =\|X\|+\left\|X^{\prime}\right\| .
\end{aligned}
$$

ii) Let $\mathcal{X}+\mathcal{X}^{\prime}=\overline{(I, J)}$. If $\overline{(I, J)}=\overline{(K, 0)}$ then $K+J=I$ and

$$
\begin{aligned}
\left\|\chi+X^{\prime}\right\| & =\|\overline{(K, 0)}\| \\
& =l(K)+|c(K)| \\
& =l(I)-l(J)+|c(I)-c(J)|
\end{aligned}
$$


that is

$$
\begin{aligned}
\left\|\chi+X^{\prime}\right\| & \leq l(I)+|c(I)|-l(J)+|c(J)| \\
& \leq l(I)+|c(I)|+l(J)+|c(J)| \\
& =\|X\|+\left\|X^{\prime}\right\| .
\end{aligned}
$$

So we have a norm on $\overline{\mathbb{I R}}$.

Theorem 4. The normed vector space $\overline{\mathbb{I R}}$ is a Banach space.

Proof. In fact, all the norms on $\mathbb{R}^{2}$ are equivalent and $\mathbb{R}^{2}$ is a Banach space for any norm. The vector space $\overline{\mathbb{I}}$ is then isomorphic to $\mathbb{R}^{2}$. Thus, it is complete.

\section{Remarks.}

1. To define the topology of the normed space $\overline{\mathbb{R}}$, it is sufficient to describe the $\varepsilon$-neighborhood of any point $\chi_{0} \in \overline{\mathbb{I R}}$ for $\varepsilon$ a positive infinitesimal number. We can give a geometrical representation, considering $\chi_{0}=\overline{([a, b], 0)}$ represented by the point $(a, b) \in \mathbb{R}^{2}$. We assume that $\chi_{0}=\overline{([a, b], 0)}$ and $\varepsilon$ an infinitesimal real number. Let $A_{1}, \ldots, A_{4}$ the points $A_{1}=(a-\varepsilon, b-\varepsilon), A_{2}=\left(a+\frac{\varepsilon}{2}, b-\frac{\varepsilon}{2}\right), A_{3}=(a+\varepsilon, b+\varepsilon), A_{4}=\left(a-\frac{\varepsilon}{2}, b+\frac{\varepsilon}{2}\right)$. If $0<a<b$, then the $\varepsilon$-neighborhood of $\chi_{0}=\overline{([a, b], 0)}$ is represented by the parallelograms whose vertices are $A_{1}, A_{2}, A_{3}, A_{4}$.

2. We can consider another equivalent norms on $\overline{\mathbb{I R}}$. For example

$$
\|X\|=\|\backslash X\|=\max \{|x|,|y|\}
$$

where $\mathcal{X}=\overline{([x, y], 0)}$, but the initial one has a better geometrical interpretation.

\subsection{Continuity and differentiability in $\overline{\mathbb{I R}}$}

As $\overline{\mathbb{I R}}$ is a Banach space, we can describe a notion of differential function on it. Consider $X_{0}=\overline{\left(X_{0}, 0\right)}$ in $\overline{\mathbb{I R}}$. The norm $\|$.$\| defines a topology on \overline{\mathbb{I R}}$ whose a basis of neighborhoods is given by the balls $\mathcal{B}\left(X_{0}, \varepsilon\right)=\left\{X \in \overline{\mathbb{I}},\left\|X \backslash X_{0}\right\|<\varepsilon\right\}$. Let us characterize the elements of $\mathcal{B}\left(X_{0}, \varepsilon\right) \cdot X_{0}=\overline{\left(X_{0}, 0\right)}=\overline{([a, b], 0)}$.

Proposition 4. Consider $X_{0}=\overline{\left(X_{0}, 0\right)}=\overline{([a, b], 0)}$ in $\overline{\mathbb{I R}}$ and $\varepsilon \simeq 0, \varepsilon>0$. Then every element of $\mathcal{B}\left(X_{0}, \varepsilon\right)$ is of type $\mathcal{X}=\overline{(X, 0)}$ and satisfies

$$
l(X) \in B_{\mathbb{R}}\left(l\left(X_{0}\right), \varepsilon_{1}\right) \text { and } c(X) \in B_{\mathbb{R}}\left(c\left(X_{0}\right), \varepsilon_{2}\right)
$$

with $\varepsilon_{1}, \varepsilon_{2} \geq 0$ and $\varepsilon_{1}+\varepsilon_{2} \leq \varepsilon$, where $B_{\mathbb{R}}(x, a)$ is the canonical open ball in $\mathbb{R}$ of center $x$ and radius $a$. 
Proof. First case: Assume that $\chi=\overline{(X, 0)}=\overline{([x, y], 0)}$. We have

$$
\begin{aligned}
x \backslash x_{0} & =\overline{\left(X, X_{0}\right)}=\overline{([x, y],[a, b])} \\
& = \begin{cases}\overline{([x-a, y-b], 0)} & \text { if } l(X) \geq l\left(X_{0}\right) \\
\overline{(0,[a-x, b-y])} & \text { if } l(X) \leq l\left(X_{0}\right)\end{cases}
\end{aligned}
$$

If $l(X) \geq l\left(X_{0}\right)$ we have

$$
\begin{aligned}
\left\|X \backslash X_{0}\right\| & =(y-b)-(x-a)+\left|\frac{y-b+x-a}{2}\right| \\
& =l(X)-l\left(X_{0}\right)+\left|c(X)-c\left(X_{0}\right)\right| .
\end{aligned}
$$

As $l(X)-l\left(X_{0}\right) \geq 0$ and $\left|c(X)-c\left(X_{0}\right)\right| \geq 0$, each one of this term if less than $\varepsilon$. If $l(X) \leq l\left(X_{0}\right)$ we have

$$
\left\|X \backslash X_{0}\right\|=l\left(X_{0}\right)-l(X)+\left|c\left(X_{0}\right)-c(X)\right| .
$$

and we have the same result.

Second case: Consider $\mathcal{X}=\overline{(0, X)}=\overline{([x, y], 0)}$. We have

$$
x \backslash x_{0}=\overline{\left(0, X_{0}+X\right)}=\overline{([x+a, y+b])}
$$

and

$$
\left\|X \backslash X_{0}\right\|=l\left(X_{0}\right)+l(X)+\left|c\left(X_{0}\right)+c(X)\right| .
$$

In this case, we cannot have $\left\|X \backslash \chi_{0}\right\|<\varepsilon$ thus $X \notin \mathcal{B}\left(X_{0}, \varepsilon\right)$.

Definition 3. A function $f: \overline{\mathbb{I R}} \longrightarrow \overline{\mathbb{I R}}$ is continuous at $\chi_{0}$ if

$$
\forall \varepsilon>0, \exists \eta>0 \text { such that }\left\|\mathcal{X} \backslash \mathcal{X}_{0}\right\|<\eta \text { implies }\left\|f(\mathcal{X}) \backslash f\left(\mathcal{X}_{0}\right)\right\|<\varepsilon \text {. }
$$

Consider $\left(\chi_{1}, \chi_{2}\right)$ the basis of $\overline{\mathbb{I R}}$ given in Section 2 . We have

$$
f(\mathcal{X})=f_{1}(\mathcal{X}) \mathcal{X}_{1}+f_{2}(\mathcal{X}) \mathcal{X}_{2} \text { with } f_{i}: \overline{\mathbb{I R}} \longrightarrow \mathbb{R}
$$

If $f$ is continuous at $\chi_{0}$ so

$$
f(\mathcal{X}) \backslash f\left(\mathcal{X}_{0}\right)=\left(f_{1}(\mathcal{X})-f_{1}\left(\mathcal{X}_{0}\right)\right) \mathcal{X}_{1}+\left(f_{2}(\mathcal{X})-f_{2}\left(\mathcal{X}_{0}\right)\right) \chi_{2}
$$

To simplify notations let $\alpha=f_{1}(\mathcal{X})-f_{1}\left(\mathcal{X}_{0}\right)$ and $\beta=f_{2}(\mathcal{X})-f_{2}\left(\mathcal{X}_{0}\right)$. If $\left\|f(\mathcal{X}) \backslash f\left(\mathcal{X}_{0}\right)\right\|<\varepsilon$, and if we assume $f_{1}(X)-f_{1}\left(\chi_{0}\right)>0$ and $f_{2}(X)-f_{2}\left(X_{0}\right)>0$ (other cases are similar), then we have

$$
l\left(\alpha \mathcal{X}_{1}+\beta \mathcal{X}_{2}\right)=l \overline{([\beta, \alpha+\beta], 0)}<\varepsilon
$$

thus $f_{1}(\chi)-f_{1}\left(\mathcal{X}_{0}\right)<\varepsilon$. Similarly,

$$
c\left(\alpha \chi_{1}+\beta \chi_{2}\right)=c \overline{([\beta, \alpha+\beta], 0)}=\frac{\alpha}{2}+\beta<\varepsilon
$$


and this implies that $f_{2}(\mathcal{X})-f_{2}\left(\mathcal{X}_{0}\right)<\varepsilon$.

Corollary 1. $f$ is continuous at $\chi_{0}$ if and only if $f_{1}$ and $f_{2}$ are continuous at $\chi_{0}$.

Definition 4. Consider $\mathcal{X}_{0}$ in $\overline{\mathbb{I}}$ and $f: \overline{\mathbb{I R}} \longrightarrow \overline{\mathbb{I R}}$ continuous. We say that $f$ is differentiable at $\mathcal{X}_{0}$ if there exists a linear function $g: \overline{\mathbb{I R}} \longrightarrow \overline{\mathbb{I R}}$ such that

$$
\left\|f(\mathcal{X}) \backslash f\left(\mathcal{X}_{0}\right) \backslash g\left(\mathcal{X} \backslash \mathcal{X}_{0}\right)\right\|=o\left(\left\|\chi \backslash \mathcal{X}_{0}\right\|\right)
$$

\section{Examples.}

- $f(\mathcal{X})=\mathcal{X}$. This function is continuous and differentiable at any point. Its derivative is $f^{\prime}(\chi)=1$.

- $f(\chi)=\chi^{2}$. Consider $\mathcal{X}_{0}=\overline{\left(X_{0}, 0\right)}=\overline{([a, b], 0)}$ and $\mathcal{X} \in \mathcal{B}\left(X_{0}, \varepsilon\right)$. We have

$$
\begin{aligned}
\left\|x^{2} \backslash x_{0}^{2}\right\| & =\left\|\left(x \backslash x_{0}\right)\left(x+x_{0}\right)\right\| \\
& \leq\left\|x \backslash x_{0}\right\|\left\|x+x_{0}\right\| .
\end{aligned}
$$

Given $\varepsilon>0$, let $\eta=\frac{\varepsilon}{\left\|\chi+\chi_{0}\right\|}$, thus if $\left\|\chi \backslash \chi_{0}\right\|<\eta$, we have $\left\|\chi^{2} \backslash \chi_{0}^{2}\right\|<\varepsilon$ and $f$ is continuous and differentiable. It is easy to prove that $f^{\prime}(\mathcal{X})=2 \chi$ is its derivative.

- Consider $P=a_{0}+a_{1} X+\cdots+a_{n} X^{n} \in \mathbb{R}[\mathbb{X}]$. We define $f: \overline{\mathbb{R}} \longrightarrow \overline{\mathbb{R}}$ with $f(X)=a_{0} \chi_{2}+a_{1} \chi+\cdots+a_{n}^{n} \chi^{n}$ where $\chi^{n}=\chi \cdot \chi^{n-1}$. From the previous example, all monomials are continuous and differentiable, it implies that $f$ is continuous and differentiable as well.

- Consider the function $Q_{2}$ given by $Q_{2}([x, y])=\left[x^{2}, y^{2}\right]$ if $|x|<|y|$ and $Q_{2}([x, y]=$ $\left[y^{2}, x^{2}\right]$ in the other case. This function is not differentiable.

\subsection{Minimization examples}

One uses the $\mathcal{A}_{7}$ pseudo-intervals arithmetic for simple examples of inclusion function minimization with first and second order methods such as respectively fixed-step gradient descent and Newton-Raphson ones.

\subsubsection{Fixed-step gradient descent method}

Let's minimize the function $x \longmapsto x \cdot \exp (x)$ with fixed-step gradient method which belongs to the so-called gradient descent method [35]. This example is very simple but it shows that the result is garanted to be found within the final interval. One sets initial guess interval to $[-5,2]$, fixed-step of descent to $10^{-2}$, finite difference step to $10^{-3}$ and accuracy of gradient to $10^{-3}$. The results shown in Figures 6, 7) show that for any initial guess the interval width decreases to converge to real point minimum. 


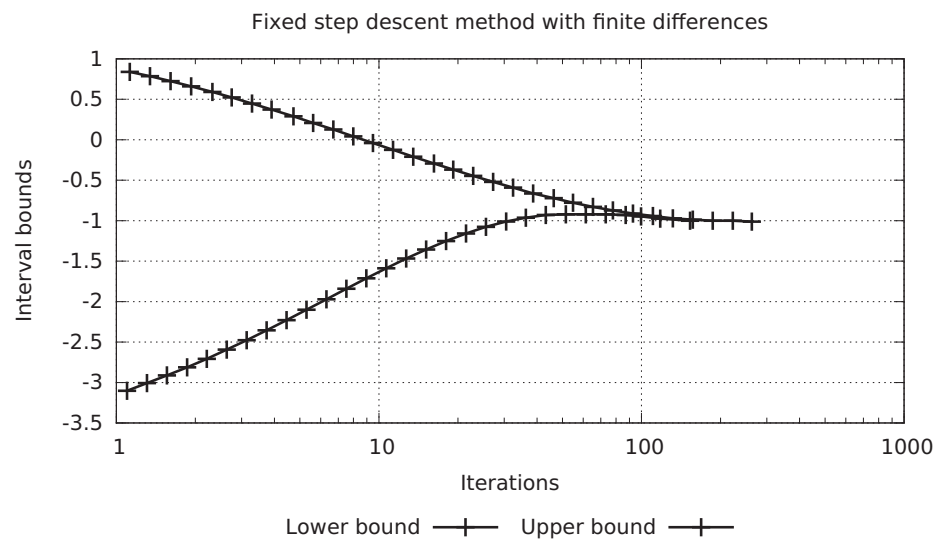

Figure 6: Convergence of the fixed-step gradient algorithm for the function $x \longmapsto x \cdot \exp (x)$ to an interval centered around $[-1,-1]=-1$.



Figure 7: Convergence of the fixed-step gradient algorithm for the derivative of the function $x \longmapsto x \cdot \exp (x)$ to an interval centered around $[0,0]=0$.

\subsubsection{Newton-Raphson method}

Let's optimize the same function $x \longmapsto x \cdot \exp (x)$ with a second order method such as the Newton-Raphson one, which is the basis of all second order methods such as Newton or quasiNewton's ones [35]. One sets initial guess interval to [0,10], finite difference step to $10^{-3}$ and accuracy of gradient to $10^{-9}$. One can state on Figures $(8,9)$ that it finds the same minimum which is an interval centered around -1 .

\section{CONCLUSION}

We have presented a better algebraic way to do calculations on intervals, called pseudo-intervals vector space or free algebra. This approach $[1,5,13]$ is done by embedding the space of intervals into a free algebra of dimension belonging to $\{4,5,7\}$. It permits to obtain all the basic arithmetic 


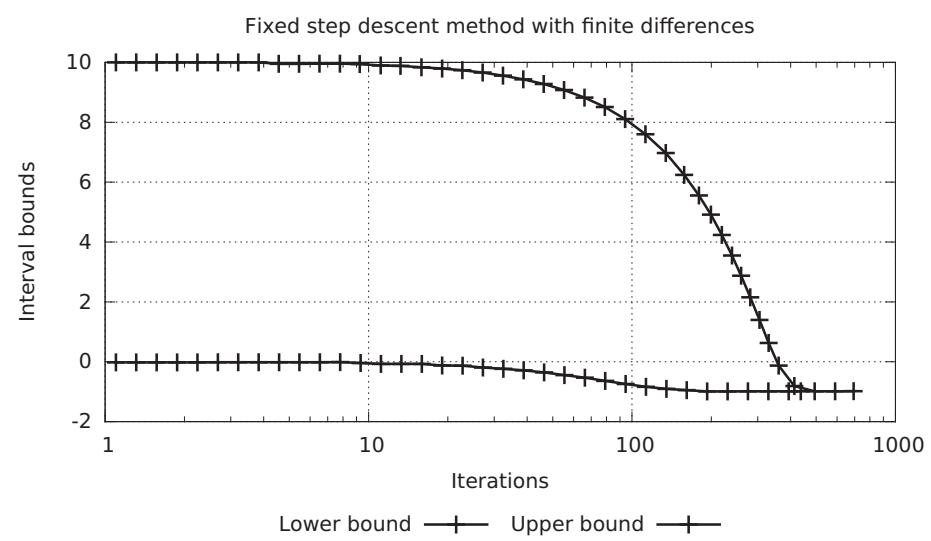

Figure 8: Convergence of the Newton-Raphson algorithm for the function $x \longmapsto x \cdot \exp (x)$ to an interval centered around $[-1,-1]=-1$.

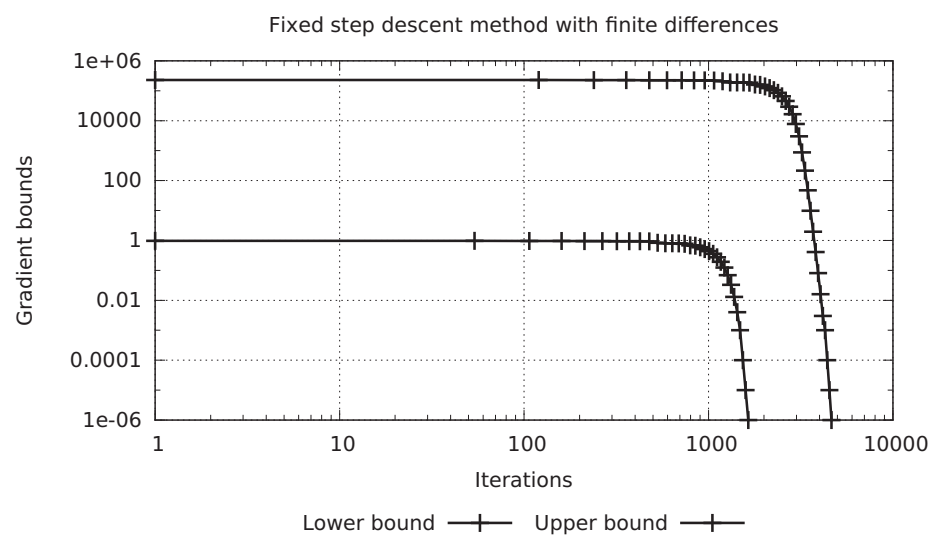

Figure 9: Convergence of the Newton-Raphson algorithm for the derivative of the function $x \longmapsto x \cdot \exp (x)$ to an interval centered around $[0,0]=0$.

operators with distributivity and associativity. We have shown that when one increases the representative algebra dimension, the multiplication result will be closer to the usual Minkowski product. It is now possible to build inclusion functions from the natural ones in a systematic way. Thus, it allows to build all algebraic operations and functions on intervals and avoids completely the wrapping effects and data dependance. One has exhibited some simple examples of applications: minimization, diagonalization and inversion of matrices which clearly state that the arithmetic is stable and that if the initial data are known with uncertainity (belonging to an interval), it is thus possible to estimate with accuracy the point solution of the problem, a real number or an interval centered around it. This pseudo-intervals arithmetic seems to be a promising and powerful computation framework in several domains of sciences and engineering, such as physics, mechanics of structure, or finance for instance. 


\section{ACKNOWLEDGEMENTS}

The author thanks Michel Gondran for useful and interesting discussions.

RESUMO. Neste artigo é proposto o uso de uma nova abordagem de aritmética intervalar, os chamados pseudo-intervalos [1, 5, 13]. Essa abordagem utiliza uma construção que é mais canônica e baseada na completude de semi-grupos em um grupo, e permite a construção de espaços vetoriais de Banach. Isso é alcançado através da imersão do espaço vetorial em uma álgebra livre de dimensão maior que 4. Desta forma é possível realizar álgebra linear e cálculo diferencial com pseudo-intervalos. Algumas aplicações numéricas para o cálculo do espectro de matrizes intervalares, inversão e minimização de funções são mostradas como exemplos.

Palavras-chave: Aritmética pseudo-intervalar, álgebra livre, inclusão de funções, álgebra linear, cálculo diferencial, otimização.

\section{REFERENCES}

[1] B. Durand, A. Kenoufi \& J.F. Osselin. System adjustments for targeted performances combining symbolic regression and set inversion. Inverse problems for science and engineering, (2013).

[2] A. Trepat, E. Gardenes \& H. Mielgo. Modal intervals: Reason and ground semantics. Lecture Notes in Computer Science, Springer-Verlag, Berlin, Heidelberg, 212 (1986), 27-35.

[3] L. Jorba, R. Calm, R. Estela, H. Mielgo, A. Trepat, E. Gardenes \& M.A. Sainz. Modal intervals. Reliable Computing, 7 (2001), 77-111.

[4] E. Gardenes. Fundamentals of sigla, an interval computing system over the completed set of intervals. Computing, 24 (1980), 161-179.

[5] N. Goze. PhD thesis, n-ary algebras and interval arithmetics. University of Haute-Alsace, (2011).

[6] A.S. Householder. The theory of matrices in numerical analysis. Dover Publications, (1975).

[7] http://www.ti3.tu-harburg.de/rump/intlab/.

[8] E. Kaucher. Ueber metrische und algebraische Eigenschaften einiger beim numerischen Rechnen auftretender Raume. Dissertation, Universitaet Karlsruhe, (1973).

[9] E. Kaucher. Algebraische erweiterungen der intervallrechnung unter erhaltung der ordnungs und verbandstrukturen computing. 1 (1977), 65-79.

[10] E. Kaucher. Ueber eigenschaften und en der anwendungsmoeglichkeiten der erweiterten intervallrechnung und des hyperbolischen fastkoerpers ueber r computing. 1 (1977), 81-94.

[11] E. Kaucher. Interval analysis in the extended interval space ir computing. 2 (1980), 33-49.

[12] R.B. Kearfott. Rigorous Global Search: Continuous Problems. Academic Publishers, (1996).

[13] A. Kenoufi. Probabilist set inversion using pseudo-intervals arithmetic. Trends in Applied and Computational Mathematics, 15(1) (2014), 97-117.

[14] O. Didrit, L. Jaulin, M. Kieffer \& E. Walter. Introduction to interval Analysis. SIAM 2009, Applied interval Analysis. Springer-Verlag, London, (2001). 
[15] S.M. Markov. Extended interval arithmetic involving infinite intervals. Mathematica Balkanica, New Series, 6(3) (1992), 269-304.

[16] S.M. Markov. On directed interval arithmetic and its applications. J. UCS, 1(7) (1995), 510-521.

[17] S.M. Markov. On the Foundations of Interval Arithmetic. Scientific Computing and Validated Numerics Akademie Verlag, Berlin, (1996).

[18] M. Markov. Isomorphic embeddings of abstract interval systems. Reliable Computing, (3) (1997), 199-207.

[19] http://maxima.sourceforge.net.

[20] R.E. Moore. PhD thesis. Standford university, (1962).

[21] R.E. Moore. Interval analysis. Prentice Hall, Englewood Cliffs, NJ, (1966).

[22] R.E. Moore. A test for existence of solutions to nonlinear systems. SIAM J. Numer. Anal., 14(4) (1977), 611-615.

[23] E. Popova, N. Dimitrova \& S.M. Markov. Extended Interval arithmetic: New Results and Applications. Computer Arithmetic and Enclosure Methods. Elsevier Sci. Publishers B.V., (1992).

[24] H.J. Ortolf. Eine verallgemeinerung der intervallarithmetik. geselschaft fuer mathematik und datenverarbeitung. Bonn, 11 (1969), 1-71.

[25] E.D. Popova. All about generalized interval distributive relations. i. complete proof of the relations. Sofia, (2000).

[26] E.D. Popova. Multiplication distributivity of proper and improper intervals. Reliable Computing, 7(2) (2001), 129-140.

[27] Yves Deville, Pascal Van Hentenryck \& Laurent Michel. Numerica: A Modelling Language for Global Optimization. MIT Press, (1997).

[28] http://www.python.org.

[29] C.T. Yang \& R.E. Moore. Interval analysis i. LMSD-285875, Lockheed Aircraft Corporation, Missiles and Space Division, Sunnyvale, California, (1959).

[30] Wayman Strother, R.E. Moore \& C.T. Yang. Interval integrals. LMSD-703073, Lockheed Aircraft Corporation, Missiles and Space Division, Sunnyvale, California, IX(4) (1960), 241-245.

[31] http://www.sagemath.org.

[32] T. Sunaga. Theory of an interval Algebra and its Application to Numerical Analysis. Number 2. RAAG Memoirs, (1958).

[33] Mieczyslaw Warmus. Calculus of approximations. Bull. Acad. Pol. Sci., C1, III(IV (5)) (1956), 253259.

[34] Mieczyslaw Warmus. Approximations and inequalities in the calculus of approximations. classification of approximate numbers. Bull. Acad. Pol. Sci. math. astr. \& phys., IX(4) (1961), 241-245.

[35] W.T. Vetterling, B.P. Flannery, W.H. Press \& S.A. Teukolsky. Numerical Recipes in C: The Art of Scientific Computing, 2nd Ed. Cambridge University Press, New York, (1992). 